\title{
Disfunción eréctil, prevalencia y factores asociados, en hombres con diabetes tipo 2, en el Eje Cafetero, Colombia, 2016-2019
}

\section{Erectile Dysfunction, Prevalence and Associated Factors, in Men with Type 2 Diabetes, in the Coffee Region, Colombia, 2016-2019}

Franklin José Espitia De La Hoz

\footnotetext{
${ }^{1}$ Ginecología y Obstetricia, Universidad Militar nueva Granada, Bogota, Colombia

2 Sexología Clínica, Universidad de Alcalá de Henares, Madrid, España

3 Uroginecología / FUCS, Hospital de San José / Unicamp, Brasil
}

\begin{abstract}
Address for correspondence Franklin José Espitia De La Hoz, MD, Carrera 12 \# 0-75, Consultorio 508 Celular: 3127436696 , Clínica del Café, Armenia, Quindío, Colombia (e-mail: espitiafranklin@hotmail.com).
\end{abstract}

Urol Colomb 2021;30:91-97.

Resumen

Palabras clave

- Diabetes Mellitus

- Hombres

- Disfunción Eréctil

- Prevalencia

- Salud Sexual
Objetivo determinar la prevalencia de la disfunción eréctil y los factores de riesgo entre los hombres con diabetes tipo 2, en el Eje Cafetero.

Métodos estudio observacional. Se realizó muestreo no probabilístico por conveniencia incorporando a 372 participantes. Se incluyeron hombres mayores de 18 años, más de un año de diagnóstico de Diabetes tipo 2 y en tratamiento periódico de su enfermedad, pertenecientes al programa de enfermedades crónicas en la consulta externa; entre 2016 y 2019, en tres instituciones universitarias de referencia ubicadas en el Eje Cafetero, Colombia. Se excluyeron analfabetos, enfermedades urológicas y los que no desearon participar. La evaluación de la salud sexual se hizo con el índice internacional de la función eréctil abreviado (IIFE-5). Variables medidas: sociodemográficas, clínicas y bioquímicas. Se aplicó estadística descriptiva.

Resultados La edad media fue de 57,59 $\pm 8,73$ años. La prevalencia de disfunción eréctil fue del $85,48 \%(n=318 / 372)$. Predominando la forma leve a moderada y severa, en un $27,33 \%$ y $26,69 \%$, respectivamente. La prevalencia de disfunción eréctil fue mayor entre los fumadores ( $O R=10,14$; IC95\%: 3,36-31,56), hipotiroideos ( $O R=5,55$; IC95\%: $2,76-8,19),(p=0,001)$ y diagnóstico de la diabetes ${ }^{3} 15$ años $(O R=3,27 ;$ IC95\%: $1,77-5,82)$.

Conclusiones la disfunción eréctil representa una complicación común, con una alta prevalencia entre los hombres con diabetes tipo 2; la cual es superior entre los fumadores, hipotiroideos y mayor tiempo de padecer la diabetes, donde se observa la más alta severidad. received

July 23, 2020

accepted

October 10, 2020

published online

March 29, 2021
DOI https://doi.org/

$10.1055 / \mathrm{s}-0040-1721333$

ISSN 0120-789X.

e ISSN 2027-0119.
(C) 2021. Sociedad Colombiana de Urología. All rights reserved. This is an open access article published by Thieme under the terms of the Creative Commons Attribution-NonDerivative-NonCommercial-License, permitting copying and reproduction so long as the original work is given appropriate credit. Contents may not be used for commercial purposes, or adapted, remixed, transformed or built upon. (https://creativecommons.org/ licenses/by-nc-nd/4.0/)

Thieme Revinter Publicações Ltda., Rua do Matoso 170, Rio de Janeiro, RJ, CEP 20270-135, Brazil 


\begin{abstract}
Keywords

- diabetes mellitus

- men

- erectile dysfunction

- prevalence

- sexual health

Objective to determine the prevalence of erectile dysfunction and the risk factors among men with type 2 diabetes, in the Coffee Region.

Methods observational study. Non-probability convenience sampling was carried out, incorporating 372 participants. Men older than 18 years, more than a year of diagnosis of type 2 diabetes and in periodic treatment of their disease, belonging to the chronic diseases program in the outpatient clinic were included; between 2016 and 2019, in three leading university institutions located in the Coffee Region, Colombia. Illiterate women, urological diseases and those who did not want to participate were excluded. The evaluation of sexual health was made with the abbreviated international index of erectile function (IIFE-5). Variables measured: socio-demographic, clinical and biochemical. Descriptive statistics were applied.

Results The mean age was $57.59 \pm 8.73$ years. The prevalence of erectile dysfunction was $85.48 \%(n=318 / 372)$. The mild to moderate and severe forms predominate, in $27.33 \%$ and $26.69 \%$, respectively. The prevalence of erectile dysfunction was higher among smokers $(\mathrm{OR}=10.14 ; 95 \% \mathrm{Cl}: 3.36-31.56)$, hypothyroid $(\mathrm{OR}=5.55 ; 95 \% \mathrm{Cl}$ : 2.76-8.19), $(\mathrm{p}=0.001)$ and diagnosis of diabetes $\geq 15$ years $(\mathrm{OR}=3.27 ; 95 \% \mathrm{Cl}: 1.77$ 5.82).

Conclusions erectile dysfunction represents a common complication, with a high prevalence among men with type 2 diabetes; which is higher among smokers, hypothyroid and longer suffering from diabetes, where the highest severity is observed.
\end{abstract}

\section{Introducción}

La disfunción eréctil (DE) se define como la incapacidad para lograr y/o mantener una erección, lo suficientemente rígida y duradera, para tener una relación sexual satisfactoria. ${ }^{1,2}$

En el Estudio de Envejecimiento Masculino de Massachusetts (en inglés: Massachusetts Male Aging Study / MMAS), realizado entre 1987 y 1989, se reportó una prevalencia de DE del $10 \%$ en los hombres entre 40 y 70 años, y del 52\% en los mayores de 70 años. ${ }^{3}$ Por su parte Lauman y col., ${ }^{4}$ mencionó una prevalencia del $5 \%$ en una población de 1.400 hombres, con edades entre 18 y 59 años.

En Latinoamérica, el estudio Disfunción Eréctil en el Norte de Sudamérica (DENSA), el cual incluyó países como Venezuela, Colombia y Ecuador, en donde se estudió una población de hombres mayores de 40 años, se reveló que la tasa de prevalencia general ajustada por edad, para cualquier grado de disfunción eréctil, era del 53,4\%. En Colombia, fue del $52,8 \%$, Ecuador del 52,1\% y Venezuela del 55,2\%; concluye que más de la mitad de los hombres mayores de 40 años de edad, en esa región, padecen algún grado de alteración en la erección. ${ }^{5}$ En Colombia, Espitia y col., ${ }^{2}$ en una cohorte de 1.902 hombres, con edad promedio de 48,6 \pm 6,9 años, reveló una prevalencia de disfunción eréctil en la población general del 47,63\%, y en la edad productiva del $39,1 \%$.

Se estima que la prevalencia mundial de la $\mathrm{DE}$, se duplicará de 152 millones de hombres, en 1995, a 322 millones en el $2025 .{ }^{6}$

El origen de la DE puede ser orgánico, psicológico, hormonal, psicosocial o de pareja ${ }^{2}$; no obstante, los principales factores de riesgo suelen ser: obesidad, tabaquismo y comorbilidades (diabetes mellitus, HTA, dislipidemia, cardiopatías, depresión / ansiedad, estrés, cáncer, etc.). ${ }^{7}$ Alrededor del $25 \%$ de los hombres con DE, tienen una causa medicamentosa (inhibidores selectivos de la recaptación de serotonina, diuréticos tiazídicos, bloqueadores simpáticos, etc.), así como el déficit de testosterona, hiperprolactinemia, hipo e hipertiroidismo. $^{8}$

En los hombres con diabetes mellitus tipo 2 (DM2), se estima que la DE oscila entre $40 \%$ y $50 \%$, alcanzando $70 \%$ a $80 \%$ en países como México, siendo mucho mayor la prevalencia en aquellos individuos con pobre control de la enfermedad. ${ }^{9,10}$

El diagnóstico de la DE incluye el uso de cuestionarios autoadministrados y validados, los cuales se utilizan de forma individual. Ellos, de manera cuantitativa y fiable, pueden clasificar la disfunción eréctil.2,11 El IIEF-5 se caracteriza porque tiene óptimas propiedades para detectar la presencia y establecer la severidad de la DE. ${ }^{2}$

En Colombia existen pocos estudios acerca de la evaluación de las disfunciones sexuales en hombres, ${ }^{2}$ especialmente en la población de diabéticos. Lo cual hace necesario, y casi obligatorio, evaluar la influencia de esa patología en la función sexual de los hombres en nuestro medio. Por ello, el objetivo del presente estudio, consistió en determinar la prevalencia y los factores de riesgo entre los hombres con diabetes tipo 2, así como la puntuación del índice internacional de la función eréctil (IIFE-5) en hombres diabéticos en el Eje Cafetero (Colombia). 


\section{Métodos}

\section{Diseño y población}

Estudio observacional descriptivo de corte transversal, al que ingresaron hombres mayores de 18 años, más de un año de diagnóstico de la Diabetes tipo 2 y en tratamiento periódico de la enfermedad, pertenecientes al programa de enfermedades crónicas en la consulta externa; entre el 01 agosto de 2016 y el 30 de septiembre de 2019, en tres instituciones universitarias de referencia ubicadas en el Eje Cafetero, Colombia. Se excluyeron analfabetos, enfermedades urológicas (prostatitis, hiperplasia prostática y prostatectomía por estar asociadas a la aparición de disfunción eréctil) y los que no desearon participar. Se realizó un muestreo por conveniencia a partir del universo de pacientes atendidos en las instituciones participantes durante el periodo de estudio. No se realizó el cálculo de tamaño de la muestra.

\section{Procedimiento}

Uno de los investigadores de cada centro participante, identificó a los hombres diabéticos tipo 2 asistentes al programa de enfermedades crónicas, en el periodo de estudio. A partir de ahí tres médicos diplomados en sexología, verificaban los criterios de elegibilidad, y hacían su labor para reclutar al paciente. Al momento de que el participante firmaba el consentimiento informado para su ingreso en la investigación, inmediatamente se le hacía la entrevista clínica y el personal de enfermería, experta en recolección de datos, revisaba y extraía la información de las historias clínicas y completaba los datos socio-demográficos, clínicos, bioquímicos, antecedentes personales y hábitos. La salud sexual se evaluó mediante el índice internacional de la función eréctil abreviado (IIFE-5), el cual se aplicó mediante interrogatorio directo por parte del médico diplomado en sexología, y perteneciente al equipo investigador, para determinar la presencia de disfunción eréctil.

El índice internacional de la función eréctil abreviado $($ IIFE-5) 2,12 es un cuestionario multidimensional, autoadministrado, psicométricamente válido, de auto aplicación (minimizando el sesgo de deseabilidad social de la heteroaplicación), confiable, estandarizado y ampliamente utilizado para el diagnóstico y clasificación de la gravedad de la disfunción eréctil; está conformado por 5 preguntas dirigidas a conocer el desempeño sexual del individuo, y a su vez, cada pregunta tiene 5 respuestas posibles. El rango de cada respuesta tiene un puntaje que varía de 1 a 5 . La puntuación es mayor cuanto mejor es el desempeño sexual y la satisfacción del individuo. El rango total de la puntuación del IIFE-5 va desde 5 hasta 25 (Anexo 1). Se considera como sano el hombre con un puntaje mayor o igual a 22 puntos, y disfunción eréctil cuando la puntuación es inferior. ${ }^{12,13}$

\section{Variables medidas}

Socio-demográficas (edad, raza, escolaridad, estrato socioeconómico, estado civil, ocupación, afiliación al sistema general de seguridad social en salud, condición espiritual o religiosa, área de residencia); hábitos (tabaquismo, ingesta de alcohol, consumo de sustancias psicoactivas, cafeína y sedentarismo); antecedentes personales; variables de comportamiento sexual (orientación sexual, edad de la primera relación sexual, frecuencia promedio de relaciones sexuales semanales, número de parejas sexuales, tiempo de convivencia en pareja, antecedente de abuso sexual o violencia sexual en el matrimonio, pareja con disfunción sexual e infidelidad) y las bioquímicas (TSH, HbA1C, glucemia, perfil lipídico). Se indagaron además las preguntas del IIFE-5.

La información se digitó en Microsoft Excel 2010 y se aplicó validación de datos en las celdas para evitar errores de digitación.

\section{Análisis estadístico}

Se efectuó mediante el programa SPSS versión $20.0{ }^{\circledR}$. Se utilizó estadística descriptiva. Se usó regresión logística binaria para calcular los valores $p$ de los Odds Ratio (OR) y el 95\% de los intervalos de confianza (IC95\%) para explicar el evento donde era necesario. Se efectuó la prueba t de Student para muestras relacionadas. Se describió la prevalencia de disfunción eréctil (número de pacientes con disfunción eréctil confirmada / total de pacientes que ingresaron).

\section{Consideraciones éticas}

En la elaboración de este estudio, se cumplieron las normas actuales de buena práctica clínica y fueron secundados los principios éticos de la declaración de Helsinki y la Resolución 8430 de 1993 del ministerio de Salud de Colombia. Se garantizó la confidencialidad de la información tanto de las pacientes como del equipo de investigación.

\section{Resultados}

Se reclutaron 495 hombres que tenían Diabetes tipo 2, de los cuales, un total de 45 no cumplían los criterios de inclusión; en 33 de ellos, no se realizaron los exámenes bioquímicos completos; 27 no completaron las variables de comportamiento sexual y 18 no aceptaron participar, lo que dio como resultado una población final de participantes de 372 .

En cuanto a las características socio-demográficas de los participantes, se observó una edad media de 57,59 $\pm 8,73$ años, mediana de 54 años (rango entre 21 y 75); en su mayoría pertenecientes al régimen contributivo (89,51\%), en el sistema de seguridad social en Colombia; estrato socioeconómico medio (73,38\%), hispánicos (69,35\%), católicos $(91,12 \%)$ y procedentes del área urbana $(81,45 \%)$ (-Tabla 1).

En relación a los antecedentes personales, se detectó una significativa presencia de enfermedades cardiovasculares $(47,84 \%)$ seguidas de la depresión / ansiedad $(44,62 \%)$ y las dislipidemias (40,86\%) (-Tabla 2).

El tiempo promedio de diagnóstico de la Diabetes tipo 2 fue de 11,43 \pm 7,29 años. Al momento del muestreo el 78,22\% $(\mathrm{n}=291 / 372)$ de los participantes se encontraban con un adecuado control glucémico. 
Tabla 1 Características socio-demográficas en hombres diabéticos con disfunción eréctil, Eje Cafetero, Colombia, 2016-2019

\begin{tabular}{|l|l|}
\hline Variable y categorías & $\mathbf{n} /$ proporción (\%) \\
\hline Edad: $X \pm D E$ años & $57,59 \pm 8,73$ \\
\hline Edad de la pareja: $X \pm D E$ & $50,79 \pm 8,46$ \\
\hline Peso: $X \pm D E ~ K g$ & $83,76 \pm 8,79$ \\
\hline Talla: $X \pm$ DE Cms & $168,93 \pm 5,49$ \\
\hline IMC: $X \pm$ DE & $29,43 \pm 7,59$ \\
\hline Estado civil & $156(41,93 \%)$ \\
\hline Casados & $135(36,29 \%)$ \\
\hline Unión libre & $57(15,32 \%)$ \\
\hline Solteros & $24(6,45 \%)$ \\
\hline Divorciados & \multicolumn{2}{|l}{} \\
\hline Escolaridad & $47(12,63 \%)$ \\
\hline Primaria & $143(38,44 \%)$ \\
\hline Secundaria & $79(21,23 \%)$ \\
\hline Técnicos & $103(27,68 \%)$ \\
\hline Profesionales & $210(56,45 \%)$ \\
\hline Ocupación & $102(27,41 \%)$ \\
\hline Empleados & $27(7,25 \%)$ \\
\hline Trabajadores independientes & \\
\hline Desempleados & \\
\hline Pensionados & \\
\hline
\end{tabular}

Tabla 2 Enfermedades crónicas en hombres diabéticos con disfunción eréctil, Eje Cafetero, Colombia, 2016-2019

\begin{tabular}{|l|l|}
\hline Patología & Prevalencia \\
\hline Artritis reumatoide & $3,49 \%$ \\
\hline Artrosis & $6,98 \%$ \\
\hline Cáncer & $22,84 \%$ \\
\hline Depresión / ansiedad & $44,62 \%$ \\
\hline Dislipidemia & $40,86 \%$ \\
\hline Enfermedad cardiovascular & $47,84 \%$ \\
\hline Enfermedad de Parkinson & $2,95 \%$ \\
\hline Hipotiroidismo & $14,24 \%$ \\
\hline HTA & $39,78 \%$ \\
\hline Prostatismo & $23,92 \%$ \\
\hline Neumopatías crónicas & $6,18 \%$ \\
\hline Osteoporosis & $9,13 \%$ \\
\hline
\end{tabular}

El Índice de masa corporal (IMC) fue de 29,43 $\pm 7,59$, el promedio de la glicemia en ayunas -en el total de participantes- fue de $97,53 \pm 13,53 \mathrm{mg} / \mathrm{dl}$. La media en la cifra de la hemoglobina glicada (HbA1c) fue de 7,59 $\pm 2,46$. El promedio de la TSH y tiroxina libre fue 3,69 $\mathrm{mIU} / \mathrm{L} \pm 2,76 \mathrm{y}$
$16,29 \pm 4,86 \mathrm{pmol} / \mathrm{L}$, respectivamente. La media de los Triglicéridos fue $186 \pm 54,63 \mathrm{mg} / \mathrm{dl}$, colesterol total $228 \pm 49,86 \mathrm{mg} / \mathrm{dl}, \quad$ Colesterol HDL $57 \pm 13,83 \mathrm{mg} / \mathrm{dl}$, Colesterol LDL $137,76 \pm 41,79 \mathrm{mg} / \mathrm{dl}$, Colesterol VLDL $34,53 \pm 13,83 \mathrm{mg} / \mathrm{dl}$. Los índices cLDL/cHDL 2,58 $\pm 1,14 \mathrm{y}$ colesterol total/cHDL $4,35 \pm 2,13$.

En relación a las variables de comportamiento sexual, la orientación sexual es de predominio heterosexual (88,97\%); la edad de la primera relación sexual arrojó una media $16,89 \pm 1,83$ años, con una mediana de 15 años (rango entre 9 y 21); la frecuencia promedio de relaciones sexuales semanales, determinó una mediana de 3 (rango entre $0 \mathrm{y}$ 6); el número de parejas sexuales reportó una mediana de 9 (rango entre $1 \mathrm{y}^{3} 30$ ); y el tiempo de convivencia en pareja alcanzó una media 13,56 $\pm 7,29$ años. El antecedente de abuso sexual reportó un $9,94 \%$, con un $12,36 \%$ de violencia sexual en el matrimonio asociado a su disfunción eréctil. El 27,15\% afirmó que la pareja presentaba alguna disfunción sexual, mientras que el $17,47 \%$ reconoció que la pareja le había sido infiel, por su parte el 32,79\% afirmó haber sido infiel por lo menos en alguna ocasión.

Respecto a la prevalencia de disfunción eréctil, ella fue del $85,48 \%(n=318 / 372)$ en la población total. Predominando la forma leve a moderada con el $27,33 \%$ seguida de la severa (26,69\%), la moderada (22,62\%) y por último la leve (22,3\%). En esa población, el 34,27\% afirmaron que su médico tratante había evaluado la sexualidad en la consulta; sin embargo, únicamente el 18,55\% de la población total, recibía algún tipo de tratamiento.

La puntuación del IIFE-5, en la población total de los 372 hombres participantes, arrojó un promedio de 14,46 $\pm 5,79$ puntos (con un mínimo de 5,56 y un máximo de 22,53); frente al $12,63 \pm 4,86$ puntos (con un mínimo de 5,56 y un máximo de 21,36$)$ de la población afectada de disfunción eréctil $(n=318 / 372)$.

En la distribución por grupos de edad, se observó que en el grupo de 18 - 29 años, la disfunción eréctil estuvo presente en el 5,34\%, frente al 77,66\% en los hombres ${ }^{3} 50$ años (-Tabla 3).

Respecto a los hábitos, el tabaquismo estuvo presente en el $20,96 \%$ de la población total $(n=70 / 372)$; la ingesta de alcohol se reportó en el 83,87\% ( $n=312 / 372)$; el consumo de sustancias psicoactivas se encontró en el 4,83\% ( $n=18 / 372)$ y la ingesta de cafeína se detectó en el 90,33\% ( $\mathrm{n}=336 / 372)$. El $16,39 \%$ ( $n=61 / 372$ ) consumía alcohol y fumaba; en ese grupo la disfunción eréctil alcanzó el $86,88 \%$. El 24,73\% $(n=92 / 372)$ consumía sustancias psicoactivas, alcohol y fumaba; en ese grupo la disfunción eréctil se hizo presente en el $90,21 \%$. El sedentarismo se encontró en el 58,06\% $(n=216 / 372)$.

Los hombres con HbA1c: $>6,9 \%$ fueron los que presentaron más disfunción eréctil y mayor grado de severidad ( $O R=2,79$; IC95\%: 1,56-5,49) en comparación con los que tenían una HbA1c: $<6,9 \%, \quad(p=0,001)$. El $35,73 \%$ de los participantes que no eran hipotiroideos, reportaron algún grado de disfunción eréctil en comparación a los hipotiroideos, los que la presentaron en el 64,15\% (OR = 5,55; IC95\%: 2,76-8,19), $(\mathrm{p}=0,001)$. 
Tabla 3 Prevalencia de disfunción eréctil por edades, en hombres diabéticos, Eje Cafetero, Colombia, 2016-2019

\begin{tabular}{|l|l|l|l|l|l|l|}
\hline Edad en años & Población & Prevalencia \% & Leve & Leve a moderada & Moderada & Severa \\
\hline 18 a 29 & 17 & 5,34 & 2,2 & 1,57 & 0,94 & 0,62 \\
\hline 30 a 39 & 23 & 7,23 & 2,51 & 1,88 & 1,57 & 1,25 \\
\hline 40 a 49 & 31 & 9,74 & 3,45 & 2,83 & 2,2 & 1,25 \\
\hline 50 a 59 & 88 & 27,67 & 4,4 & 8,17 & 5,34 & 9,74 \\
\hline 60 a 69 & 103 & 32,38 & 6,91 & 9,11 & 7,86 & 8,49 \\
\hline$\geq 70$ & 56 & 17,61 & 2,83 & 3,77 & 4,71 & 5,34 \\
\hline Total & 318 & $100 \%$ & 22,3 & 27,33 & 22,62 & 26,69 \\
\hline
\end{tabular}

El grupo de hombres diabéticos con disfunción eréctil severa, tenían una evolución ${ }^{3}$ a partir de los 15 años de habérsele diagnosticado la Diabetes, representando el 10,95\% de la población total de participantes, alcanzando la duración de la diabetes, un aumento en el riesgo de disfunción eréctil (DE) $(\mathrm{OR}=3,27$; IC95\%: $1,77-5,82)$, en comparación con los que tenían menos tiempo de habérseles diagnosticado $(\mathrm{p}=0,001)$. De igual forma, se encontró asociación entre los ${ }^{3} 50$ años con relación a los menores ( $\mathrm{OR}=1,17$; IC95\%: 1,02-1,59); casados respecto a los solteros ( $\mathrm{OR}=2,52$; IC95\%: 1,26-5,13), $(\mathrm{p}=0,001)$; nivel escolar primario ( $\mathrm{OR}=1,23$; IC95\%: $1,08-1,74),(\mathrm{p}=0,001)$ y con complicaciones de la Diabetes (OR $=2,88$; IC95\%: 1,17 $7,26),(p=0,001)$. Al evaluar los fumadores frente a los que no fumaban, persistió siendo mayor tanto la frecuencia como la severidad de la $\mathrm{DE}(\mathrm{OR}=10,14$; IC95\%: 3,36-31,56).

\section{Discusión}

Se encontró una prevalencia de disfunción eréctil en hombres diabéticos del Eje Cafetero del 85,48\%; predominando la forma leve a moderada en un $27,33 \%$ seguida de la severa con el 26,69\%; en donde la puntuación del IIFE-5 arrojó un promedio de 14,46 $\pm 5,79$ puntos en la población total de hombres participantes.

Al comparar nuestros resultados con un estudio realizado en México por Ramírez-Ramos y col., ${ }^{14}$ en una muestra de 95 participantes mayores de 40 años, y otro en el Perú por Manrique y col., ${ }^{15}$ en una muestra de 100 participantes, ellos reportan una prevalencia parecida a la expuesta en el presente estudio, con $89,5 \%$ y $86 \%$, respectivamente. El tipo de población estudiada podría explicar la significativa prevalencia, ya que nuestro estudio incluyó hombres con similares características socio-demográficas a la de las otras investigaciones. Sin embargo, los hallazgos son inferiores a los informados por Venegas y col.., ${ }^{16}$ en Chile, con una prevalencia del $51,1 \%$ en una muestra de 1,447 hombres cuyas edades oscilaban entre 40 y 92 años.

Respecto a la puntuación del IIFE-5, nuestros resultados son inferiores a los informados por López-Morales y col.., ${ }^{17}$ en México, en una muestra de 120 participantes, quienes reportan una media, en el puntaje del IIFE-5, de 21,55 $\pm 6,48$ puntos. La razón se debe a que nuestros pacientes tenían una cifra de HbA1C con una media mucha mayor y también por tratarse de una población de más edad.

Un estudio realizado en España [(The DIVA (DIabetes and VAscular disease) study)], ${ }^{18}$ donde se incluyeron un total de 2.444 pacientes con diabetes tipo 2 (56\% hombres; edad media 65,2 años); concluye que el $40 \%$ tenían DE. Los factores implicados en dicha DE estarían relacionados con el control glucémico y lipémico deficiente ( $\mathrm{p}<0,05$ en ambos casos), más frecuentes entre los pacientes con DE grave; siendo similar a lo sustentado en nuestros resultados; a la luz de los reportes del presente estudio, tanto la enfermedad cardiovascular como la disfunción renal $(p<0,001)$ fueron más frecuentes entre los pacientes con DE grave, no obstante, nosotros encontramos en nuestra población, características determinantes, no modificables, como lo son los antecedentes socio-demográficos.

Al igual que en nuestro estudio, López y col., ${ }^{17}$ concluyen que la prevalencia de la $D E$ es superior a medida que se incrementa la edad del paciente, así como cuando los niveles de la HbA1C son mucho más altos, siendo usual la mayor severidad de la DE; en lo cual concordamos con Fedele y col., ${ }^{19}$ quien afirma que la severidad de la DE está correlacionada con el control glucémico, la duración de la enfermedad y la presencia de complicaciones inducidas por la diabetes.

Los profesionales de la salud deben reconocer que el inadecuado manejo de la diabetes tipo 2 , así como la falta de detección de sus complicaciones, pueden tener serias y negativas repercusiones en la salud sexual de los pacientes, pudiendo impactar de forma negativa a nivel psicológico y emocional; por lo tanto, urge un adecuado manejo de la patología.

Algunos investigadores ${ }^{20}$ han demostrado que la DE, en pacientes con diabetes tipo 2 , está relacionada con la percepción del estado de salud; asesoría y orientación que no evaluamos ni visualizamos en la presente investigación; por lo tanto, consideramos que el equipo de salud de pacientes crónicos, debe ofrecer oportuna asistencia a la hora de evaluar los conflictos sexuales que pudiera estar presentando esa población.

En nuestro estudio, el $34,27 \%$ de los participantes afirmaron que su médico tratante había evaluado la sexualidad durante la consulta, proporción similar a lo 
encontrado en un estudio danés, ${ }^{21}$ el cual mostró que solo el $33 \%$ de los hombres con diabetes tipo 2 informaron que su médico había mencionado la sexualidad en la consulta.

Un meta-análisis de 12 estudios, recientemente demostró que la DE está asociada con un incremento sustancial en el riesgo de eventos cardiovasculares, enfermedad coronaria y enfermedades vasculares periféricas ${ }^{22}$; por lo tanto, es perentorio recurrir al uso de un cuestionario de fácil aplicación para detectar la DE, no sólo como un trastorno propio de la función sexual del hombre, sino también como un marcador de riesgo cardiovascular independiente, tal como lo ha descrito Guirao y col., ${ }^{23}$ en Murcia (España).

Muchos autores afirman que la DE puede preceder (entre $3 \mathrm{y}$ 4 años) en aparición a la enfermedad cardiovascular; antelación atribuible al menor tamaño de las arterias peneanas (1-2 mm de diámetro) en comparación con el de las arterias coronarias $(3-4 \mathrm{~mm})^{24,25}$; lo que se convierte en razón de peso para evaluar esa condición en los hombres diabéticos.

La principal limitación del estudio es el hecho de estar basado en una población de pacientes que asistían a centros de atención de enfermedades crónicas, como la diabetes; lo cual puede diferir de los hombres que no asisten a esos programas, y que no son evaluados de forma integral, como sí lo hacen los médicos entrenados en esos programas. Por otra parte, el haber excluido a los analfabetos, puede minimizar el impacto socio-demográfico de esa población participante. De igual manera sucede con los hombres con enfermedades urológicas, por ser pacientes que pudieran requerir mayor intervención, por eso podría haber un sesgo de exclusión de hombres más severamente enfermos, y ser representativos de la población general. El elevado porcentaje de participantes del régimen contributivo, excluye la posibilidad de evaluar a los hombres de alta vulnerabilidad socio-económica, lo que puede dificultar la extrapolación de los resultados a la población en general. Como fortaleza tenemos el significativo número de participantes, así como de ser la primera investigación realizada en Colombia con las variables estudiadas. A la luz de los presentes resultados, esperamos que los profesionales de la salud, encargados del seguimiento y manejo de las patologías crónicas como la diabetes, se preocupen en evaluar y tratar la disfunción eréctil como un factor más de la calidad de vida.

\section{Conclusiones}

La disfunción eréctil es una frecuente complicación en hombres diabéticos del Eje Cafetero, sobrepasando las 4/5 partes de la población de participantes; predominando la forma leve a moderada $(27,33 \%$ ) y la severa $(26,69 \%)$, lo que la convierte en un problema de salud pública, y obliga a la capacitación del personal sanitario que atiende a esa población, a fin de hacer un temprano diagnóstico y oportuno tratamiento.

\section{Financiación}

La presente investigación es producto del esfuerzo económico del autor, cuyos recursos fueron obtenidos de sus turnos de obstetricia.
Conflicto de intereses

Los autores declaran no tener ningún conflicto de intereses.

\section{Agradecimientos}

A todo el personal de Hathor, Clínica Sexológica, por su constante trabajo y dedicación en la búsqueda de llevar a feliz término, nuestros proyectos de investigación; así como al equipo de bioestadística y epidemiólogos que nos asesoraron.

\section{Referencias}

1 Wang J, Zhou Y, Dai $\mathrm{H}$, et al. The safety and efficacy of acupuncture for erectile dysfunction: A network meta-analysis. Medicine (Baltimore) 2019;98(02):e14089. Doi: 10.1097/MD. 0000000000014089

2 Espitia-De La Hoz. Prevalencia de disfunción eréctil en hombres del Quindío, y factores de riesgo asociados. Urol Colomb 2019;28 (02):169-176. Doi: 10.1055/s-0038-1656515

3 Feldman HA, Johannes CB, Araújo AB, Mohr BA, Longcope C, McKinlay JB. Low dehydroepiandrosterone and ischemic heart disease in middle-aged men: prospective results from the Massachusetts Male Aging Study. Am J Epidemiol 2001;153 (01):79-89

4 Laumann EO, Paik A, Rosen RC. Sexual dysfunction in the United States: prevalence and predictors. JAMA 1999;281(06):537-544. Doi: $10.1001 /$ jama.281.6.537

5 Morillo LE, Díaz J, Estevez E, et al. Prevalence of erectile dysfunction in Colombia, Ecuador, and Venezuela: a population-based study (DENSA). Int J Impot Res 2002;14 (Suppl 2):S10-S18. Doi: 10.1038/sj.ijir.3900893

6 Ayta IA, McKinlay JB, Krane RJ. The likely worldwide increase in erectile dysfunction between 1995 and 2025 and some possible policy consequences. BJU Int 1999;84(01):50-56. Doi: 10.1046/ j.1464-410x.1999.00142.x

7 Selvin E, Burnett AL, Platz EA. Prevalence and risk factors for erectile dysfunction in the US. Am J Med 2007;120(02):151-157. Doi: 10.1016/j.amjmed.2006.06.010

8 Wein AJ, Van Arsdalen KN. Drug-induced male sexual dysfunction. Urol Clin North Am 1988;15(01):23-31

9 Manrique H, Cornejo P, Arismendiz L, Pamo A. Características clínicas y prevalencia de disfunción eréctil en pacientes con diabetes mellitus 2. Rev. Soc. PerúMed Interna 2002;15(02):

10 Gómez F. Relación entre la disfunción eréctil y la enfermedad coronaria. Rev Med Costa Rica Centroamérica 2009;(590): 377-382

11 Rosen RC, Riley A, Wagner G, Osterloh IH, Kirkpatrick J, Mishra A. The international index of erectile function (IIEF): a multidimensional scale for assessment of erectile dysfunction. Urology 1997;49(06):822-830. Doi: 10.1016/s0090-4295(97) 00238-0

12 Rosen RC, Cappelleri JC, Smith MD, Lipsky J, Peña BM. Development and evaluation of an abridged, 5-item version of the International Index of Erectile Function (IIEF-5) as a diagnostic tool for erectile dysfunction. Int J Impot Res 1999;11 (06):319-326. Doi: 10.1038/sj.ijir.3900472

13 Espitia-De La Hoz FJ, Orozco-Santiago L. Eficacia de dos inhibidores de la fosfodiesterasa 5 combinados con ginkgo biloba en la disfunción eréctil en hombres con enfermedades crónicas. Revista Colombiana de Endocrinología. Diabetes Metab 2019;6(03):155-163

14 Ramírez-Ramos JK, Borboa-García CA, Delgado-Quiñones EG, Valdeolivar-Hernández MS, Cervantes-Sánchez P, HernándezCalderón J. Prevalencia de disfunción eréctil en pacientes diabéticos mayores de 40 años. Rev Med MD 2015;6(04):280-284 
15 Manrique-Hurtado H, Cornejo-Arenas P, Neyra-Arismendiz L, Pamo-Reyna AO. Características clínicas y prevalencia de disfunción eréctil en pacientes con diabetes mellitus 2. Rev. Soc. Perú. Med Interna 2002;15(02):

16 Venegas JA, Baeza R. Prevalencia de disfunción eréctil en Chile. Rev Chil de Urol 2004;69:199-202

17 López CM, Heredia E, González R, Rosales E. Disfunción eréctil en portadores de diabetes mellitus tipo 2 en edad productiva. Rev Med Chil 2013;141:1555-1559. Doi: 10.4067/S003498872013001200009

18 González-Juanatey JR, Alegría Ezquerra E, Gomis Barberá R, et al; Estudio DIVA. [Erectile dysfunction as a marker of silent cardiovascular disease in type-2 diabetic patients in Spain. The DIVA (DIabetes and VAscular disease) study]. Med Clin (Barc) 2009;132(08):291-297. Doi: 10.1016/j.medcli.2008.06.009

19 Fedele D, Coscelli C, Santeusanio F, et al. Erectile dysfunction in diabetic subjects in Italy. Gruppo Italiano Studio Deficit Erettile nei Diabetici. Diabetes Care 1998;21(11):1973-1977. Doi: 10.2337/diacare.21.11.1973

20 De Berardis G, Franciosi M, Belfiglio M, et al; Quality of Care and Outcomes in Type 2 Diabetes (QuED) Study Group. Erectile dysfunction and quality of life in type 2 diabetic patients: a serious problem too often overlooked. Diabetes Care 2002;25 (02):284-291. Doi: 10.2337/diacare.25.2.284

21 Diabetes Association Undersøgelse af rejsningsbesvær blandt diabetikere (Study of erectile dysfunction among diabetes patients). 2007. (accessed Marz 05, 2020) at: http://www. diabetes.dk/media/352262/Rejsningsbesv\%C3\%

A6r_blandt_diabetikere.pdf

22 Low WY, Ng CJ, Tan NC, Choo WY, Tan HM. Management of erectile dysfunction: barriers faced by general practitioners. Asian J Androl 2004;6(02):99-104

23 Guirao Sánchez L, García-Giralda Ruiz L, Casas Arandac I, Alfaro González JV, López Caravacaa J, Sánchez Pérez GA. Disfunción eréctil como factor de riesgo cardiovascular independiente en atención primaria. Rev Int Androl 2006;4(02):70-73. Doi: 10.1016/s1698-031x(06)73573-7

24 Montorsi P, Ravagnani PM, Galli S, et al. The artery size hypothesis: a macrovascular link between erectile dysfunction and coronary artery disease. Am J Cardiol 2005;96(12B):19M-23M. Doi: 10.1016/j.amjcard.2005.07.006

25 Tárraga López PJ, Celada A, Baquero Torres RM. Disfunción eréctil y diabetes. Medicina General 2005;76:525-526

Anexo 1 Índice Internacional de la Función Eréctil abreviado (IIEF-5)

\begin{tabular}{|c|c|c|c|c|}
\hline \multicolumn{5}{|c|}{ 1. ¿Cómo califica la confianza que tiene en poder mantener una erección? } \\
\hline $\begin{array}{l}1 \\
\text { Muy baja }\end{array}$ & $\begin{array}{l}2 \\
\text { Baja }\end{array}$ & $\begin{array}{l}3 \\
\text { Moderada }\end{array}$ & $\begin{array}{l}4 \\
\text { Alta }\end{array}$ & $\begin{array}{l}5 \\
\text { Muy alta }\end{array}$ \\
\hline \multicolumn{5}{|c|}{$\begin{array}{l}\text { 2. Cuando tiene erecciones mediante estimulación sexual, ¿con qué frecuencia sus erecciones tienen la dureza suficiente para la } \\
\text { penetración? }\end{array}$} \\
\hline $\begin{array}{l}1 \\
\text { Casi nunca }\end{array}$ & $\begin{array}{l}2 \\
\text { Pocas veces }\end{array}$ & $\begin{array}{l}3 \\
\text { Algunas veces }\end{array}$ & $\begin{array}{l}4 \\
\text { Muchas veces }\end{array}$ & $\begin{array}{l}5 \\
\text { Casi siempre }\end{array}$ \\
\hline \multicolumn{5}{|c|}{ 3. Durante las relaciones sexuales, ¿con qué frecuencia puede mantener su erección después de haber penetrado a su pareja? } \\
\hline $\begin{array}{l}1 \\
\text { Casi nunca }\end{array}$ & $\begin{array}{l}2 \\
\text { Pocas veces }\end{array}$ & $\begin{array}{l}3 \\
\text { Algunas veces }\end{array}$ & $\begin{array}{l}4 \\
\text { Muchas veces }\end{array}$ & $\begin{array}{l}5 \\
\text { Casi siempre }\end{array}$ \\
\hline \multicolumn{5}{|c|}{ 4. Durante las relaciones sexuales, ¿qué tan difícil es mantener su erección hasta el final del acto sexual? } \\
\hline $\begin{array}{l}1 \\
\text { Extremadamente difícil }\end{array}$ & $\begin{array}{l}2 \\
\text { Muy difícil }\end{array}$ & $\begin{array}{l}3 \\
\text { Difícil }\end{array}$ & $\begin{array}{l}4 \\
\text { Ligeramente difícil }\end{array}$ & $\begin{array}{l}5 \\
\text { Nada difícil }\end{array}$ \\
\hline \multicolumn{5}{|c|}{ 5. Cuando trataba de tener relaciones sexuales, ¿con qué frecuencia eran satisfactorias para usted? } \\
\hline $\begin{array}{l}1 \\
\text { Casi nunca }\end{array}$ & $\begin{array}{l}2 \\
\text { Pocas veces }\end{array}$ & $\begin{array}{l}3 \\
\text { Algunas veces }\end{array}$ & $\begin{array}{l}4 \\
\text { Muchas veces }\end{array}$ & $\begin{array}{l}5 \\
\text { Casi siempre }\end{array}$ \\
\hline \multicolumn{5}{|l|}{ Interpretación de los resultados: } \\
\hline \multicolumn{5}{|c|}{ Sumar los números que corresponden a las respuestas de las preguntas 1 a 5} \\
\hline \multicolumn{5}{|c|}{$\begin{array}{l}\text { La sumatoria de la puntuación obtenida de todos los ítems, en el IIEF-5, caracteriza la intensidad de la Disfunción Eréctil de la } \\
\text { siguiente manera: }\end{array}$} \\
\hline \multicolumn{5}{|l|}{ 22-25: Sin disfunción eréctil } \\
\hline \multicolumn{5}{|l|}{ 17-21: Disfunción eréctil leve } \\
\hline \multicolumn{5}{|c|}{ 12-16: Disfunción eréctil leve a moderada } \\
\hline \multicolumn{5}{|c|}{ 8-11: Disfunción eréctil moderada } \\
\hline 5-7: Disfunción eréctil severa & & & & \\
\hline
\end{tabular}

